\title{
$M$-IDEALS OF COMPACT OPERATORS
}

\section{Chong-Man Cho}

\begin{abstract}
Suppose $X$ is a reflexive Banach space and $Y$ is a closed subspace of a $c_{0}$-sum of finite dimensional Banach spaces. If $K(X, Y)$, the space of the compact linear operators from $X$ to $Y$, is dense in $L(X, Y)$, the space of the bounded linear operators from $X$ to $Y$, in the strong operator topology, then $K(X, Y)$ is an $M$-ideal in $L(X, Y)$.
\end{abstract}

1. Introduction. Harmand and Lima [7] proved that if $X$ is a Banach space for which $K(X)$, the space of compact operators on $X$, is an $M$ ideal in $L(X)$, the space of continuous linear operators on $X$, then there exists a net $\left\{T_{\alpha}\right\}$ in $K(X)$ such that

(i) $\left\|T_{\alpha}\right\| \leq 1$ for all $\alpha$,

(ii) $T_{\alpha} \rightarrow I_{X}$ strongly,

(iii) $T_{\alpha}^{*} \rightarrow I_{X^{*}}$ strongly,

(iv) $\left\|I_{X}-T_{\alpha}\right\| \rightarrow 1$.

Thus, if $K(X)$ is an $M$-ideal in $L(X)$ then $X$ satisfies the metric compact approximation property.

Later Cho and Johnson [3] proved that if $X$ is a closed subspace of $\left(\sum_{n=1}^{\infty} X_{n}\right)_{p}\left(\operatorname{dim} X_{n}<\infty, 1<p<\infty\right)$ which has the compact approximation property, then $K(X)$ is an $M$-ideal in $L(X)$.

Recently Werner [15] obtained the same conclusion for a closed subspace $X$ of a $c_{0}$-sum of finite dimensional Banach spaces which has the metric compact approximation property. More specifically, he proved the following.

THEOREM. If $X$ is a closed subspace of a $c_{0}$-sum of finite dimensional spaces, then the following are equivalent:

(a) $X$ has the metric compact approximation property.

(b) For each Banach space $W, K(W, X)$ is an $M$-ideal in $L(W, X)$.

(c) $K(X)$ is an $M$-ideal in $L(X)$.

Werner's proof [15] of the implication (c) $\Rightarrow$ (a) above can be used in the case of a pair of Banach spaces $X$ and $Y$ to prove that if $K(X, Y)$ is an $M$-ideal in $L(X, Y)$, then the closed unit ball of $K(X, Y)$ is dense in the closed unit ball of $L(X, Y)$ in the topology of uniform convergence on compact sets in $X$. 
The main result of this paper is Theorem 3. In Theorem 3 we will prove that if $X$ is a reflexive Banach space and $Y$ is a closed subspace of $\left(\sum Z_{i}\right)_{c_{0}}$, the $c_{0}$-sum of a family $\left\{Z_{i}: i \in I\right\}$ of finite dimensional Banach spaces, for which $K(X, Y)$ is dense in $L(X, Y)$ in the strong operator topology, then $K(X, Y)$ is an $M$-ideal in $L(X, Y)$. Thus, if either $X$ or $Y$ has the compact approximation property then $K(X, Y)$ is an $M$-ideal in $L(X, Y)$.

2. Notation and preliminaries. A closed subspace $J$ of a Banach space $X$ is said to be an $L$-summand if there exists a closed subspace $J^{\prime}$ of $X$ such that $X$ is an algebraic direct sum of $J$ and $J^{\prime}$, and also satisfies a norm condition $\left\|j+j^{\prime}\right\|=\|j\|+\left\|j^{\prime}\right\|$ for all $j \in J$ and $j^{\prime} \in J^{\prime}$. In this case we write $X=J \oplus_{1} J^{\prime}$. A closed subspace $J$ of a Banach space $X$ is called an $M$-ideal in $X$ if $J^{0}$, the annihilator of $J$ in $X^{*}$, is an $L$-summand in $X^{*}$.

If $X$ and $Y$ are Banach spaces, $L(X, Y)$ (resp. $K(X, Y)$ ) will denote the space of all bounded linear operators (resp. compact operators) from $X$ to $Y$. If $X=Y$, then we simply write $L(X)($ resp. $K(X)$ ). Unless otherwise specified, these spaces are understood to be Banach spaces with operator norm.

If $X$ is a Banach space, $B_{X}$ will denote the closed unit ball of $X$. A Banach space $X$ is said to have the compact approximation property (resp. metric compact approximation property) if the identity operator on $X$ is in the closure of $K(X)$ (resp. $B_{K(X)}$ ) with respect to the topology of uniform convergence on compact sets in $X$.

If $\left\{Z_{i}: i \in I\right\}$ is a family of Banach spaces, the $c_{0}$-sum $\left(\sum Z_{i}\right)_{c_{0}}$ of $\left\{Z_{i}\right\}$ is the Banach space of all functions $z$ on $I$ with the properties that for $i \in I, z(i) \in Z_{i}$ and for any $\varepsilon>0$ there exists a finite set $A \subseteq I$ such that $|z(i)|<\varepsilon$ for $i \in I \backslash A$. The norm on $\left(\sum Z_{i}\right)_{c_{0}}$ is the supremum norm. For a subset $A$ of $I$, the projection $P_{A}$ in $\left(\sum Z_{i}\right)_{c_{0}}$ associated with $A$ is defined by

$$
\left(P_{A} z\right)(i)= \begin{cases}z(i) & \text { if } i \in A, \\ 0 & \text { if } i \notin A \text { for } z \in\left(\sum Z_{i}\right)_{c_{0}} .\end{cases}
$$

3. $M$-ideals. Alfsen and Effros [1] and Lima [9] characterized $M$ ideals by the intersection properties of balls. In this paper we will use the following characterization of $M$-ideals due to Lima [9]: A closed subspace $J$ of a Banach space $X$ is an $M$-ideal in $X$ if and only if for any $\varepsilon>0$, for any $x \in B_{X}$ and for any $y_{i} \in B_{J}(i=1,2,3)$, there exists $y \in J$ such that $\left\|x+y_{i}-y\right\| \leq 1+\varepsilon$ for $i=1,2,3$. 
The following theorem is essentially due to Werner [15] although he restricted attention to the case $X=Y$ and the identity map on $X$.

THEOREM 1. Let $X$ and $Y$ be Banach spaces. If $K(X, Y)$ is an $M$ ideal in $L(X, Y)$, then $B_{K(X, Y)}$ is dense in $B_{L(X, Y)}$ in the topology of uniform convergence on compact sets in $X$.

Proof. Suppose $K(X, Y)$ is an $M$-ideal in $L(X, Y)$ and suppose $L(X, Y)^{*}=K(X, Y)^{\circ} \oplus_{1} J$ for a subspace $J$ of $L(X, Y)^{*}$. Then the map $\phi \rightarrow \phi+K(X, Y)^{\circ}$ defines an isometry from $J$ onto $L(X, Y)^{*} / K(X, Y)^{\circ}$ and hence the map $\left.\phi \rightarrow \phi\right|_{K(X, Y)}$ defines an isometry from $J$ onto $K(X, Y)^{*}$ via $L(X, Y)^{*} / K(X, Y)^{\circ}$.

Let $Q$ be the projection from $L(X, Y)^{*}$ onto $J$. Then $\phi \in L(X, Y)^{*}$ is in the range of $Q$ if and only if the restriction of $\phi$ to $K(X, Y)$ has the same norm as $\phi$.

If $T \in L(X, Y) \subseteq L(X, Y)^{* *}$ with $\|T\| \leq 1$, then for $\phi=\phi_{1}+\phi_{2}$ in $L(X, Y)^{*}$ with $\phi_{1} \in K(X, Y)^{\circ}$ and $\phi_{2} \in J$ we have

$$
\left(Q^{*} T\right) \phi=T Q\left(\phi_{1}+\phi_{2}\right)=T \phi_{2} .
$$

Thus $Q^{*} T \in K(X, Y)^{\circ}{ }^{\circ}=J^{*}=K(X, Y)^{* *}$.

Since $Q^{*} T \in K(X, Y)^{* *}$ and $\left\|Q^{*} T\right\| \leq 1$, by Goldstine's theorem there is a net $\left\{K_{\alpha}\right\}$ in $B_{K(X, Y)}$ such that

$$
K_{\alpha} \rightarrow Q^{*} T \text { in the weak*-topology induced by } K(X, Y)^{*} \text {. }
$$

Since for each $x \in X$ and each $y^{*} \in Y^{*}, y^{*} \otimes x$ is in the range of $Q$, we have

$$
y^{*}\left(K_{\alpha} x\right)=K_{\alpha}\left(y^{*} \otimes x\right) \rightarrow\left(Q^{*} T\right)\left(y^{*} \otimes x\right)=y^{*}(T x) .
$$

This shows that $T$ is in the closure of $B_{K(X, Y)}$ in the weak operator topology and hence in the strong operator topology.

The following theorem plays a key role in the proof of the main theorem.

THEOREM 2. Let $X$ be a reflexive Banach space and let $Y$ be a closed subspace of $Z=\left(\sum Z_{i}\right)_{c_{0}}$, the $c_{0}$-sum of a family $\left\{Z_{i}: i \in I\right\}$ of finite dimensional Banach spaces. If $K(X, Y)$ is dense in $L(X, Y)$ in the strong operator topology, then for any $T \in B_{L(X, Y)}$ there exist nets $\left\{K_{\alpha}\right\}$ in $K(X, Y)$ and $\left\{Q_{\alpha}\right\}$ in $B_{K(X, Z)}$ such that $\left\|T-Q_{\alpha}\right\| \leq\|T\|$, 
$\left\|Q_{\alpha}-K_{\alpha}\right\| \rightarrow 0$ and for any finite subset $A$ of $I$ there exists $\alpha_{0}$ such that $P_{A}\left(T-Q_{\alpha}\right)=0$ for $\alpha \geq \alpha_{0}$.

Proof. Let $T \in B_{L(X, Y)}$ and let $\left\{T_{\beta}\right\}$ be a net in $K(X, Y)$ such that $T_{\beta} \rightarrow T$ strongly. View $T$ and $T_{\beta}$ 's as operators in $L(X, Z)$. Since $P_{A} T \rightarrow T$ strongly ( $A$ ranges over the finite subsets of $I$ ), by replacing the index sets of $\left\{T_{\beta}\right\}$ and $\left\{P_{A}\right\}$ by the product directed set we have $T_{\gamma}-P_{\gamma} T \rightarrow 0$ strongly and there exists $r>0$ such that $\left\|T_{\gamma}-P_{\gamma} T\right\| \leq r$ for all $\gamma$.

We claim that $T_{\gamma}-P_{\gamma} T \rightarrow 0$ weakly in $K(X, Z) . Z^{*}=\left(\sum Z_{i}^{*}\right)_{l^{1}}$ has the metric approximation property and the Radon Nikodym property [5, p. 219]. Since $Z$ is an $M$-ideal in $Z^{* *}$ [7], $Z^{*}$ is complemented in $Z^{* * *}$ by norm one projection [7].

Thus $K(X, Z)^{*}=X^{* *} \hat{\oplus} Z^{*}=X \hat{\otimes} Z^{*}$ [5, p. 247].

If $x \otimes z^{*} \in X \hat{\otimes} Z^{*}$ and $T=\sum_{i=1}^{n} x_{i}^{*} \otimes z_{i}$ is a finite rank operator from $X$ to $Z$,

$$
\left(x \otimes z^{*}\right)(T)=\sum_{i=1}^{n} x\left(x_{i}^{*}\right) z^{*}\left(z_{i}\right)=z^{*}(T x) .
$$

Since the finite rank operators are dense in $K(X, Z)$,

$$
\left(x^{* *} \otimes z^{*}\right)\left(T_{\gamma}-P_{\gamma} T\right)=x^{* *}\left(T_{\gamma}-P_{\gamma} T\right)^{*} z^{*} \rightarrow 0 .
$$

Since $X \otimes Z^{*}$ is dense in $X \hat{\otimes} Z^{*}=K(X, Z)^{*}, T_{\gamma}-P_{\gamma} T \rightarrow 0$ weakly in $K(X, Z)$.

Since $T_{\gamma}-P_{\gamma} T \rightarrow 0$ weakly in $K(X, Z)$, there exists a net $\left\{K_{\alpha}-Q_{\alpha}\right\}$ of convex combinations of $\left\{T_{\gamma}-P_{\gamma} T\right\}$ which converges to zero in norm, where $K_{\alpha}$ is a convex combination of $T_{\gamma}$ 's and $Q_{\alpha}$ is a convex combination of $P_{\gamma} T$ 's. Moreover, we can choose the net $\left\{K_{\alpha}-Q_{\alpha}\right\}$ so that for any finite set $A$ of $I$, there exists $\alpha_{0}$ such that

$$
P_{A} Q_{\alpha}=P_{A} T \text { for all } \alpha \geq \alpha_{0} \text {. }
$$

From the construction of $Q_{\alpha}$, it is obvious that $\left\|\left(T-Q_{\alpha}\right) x\right\| \leq\|T x\|$ for all $\alpha$ and all $x \in X$.

TheOREM 3. Let $X, Y$ and $Z$ be as in Theorem 2. If $K(X, Y)$ is dense in $L(X, Y)$ in the strong operator topology, then $K(X, Y)$ is an $M$-ideal in $L(X, Y)$.

Proof. Let $S_{1}, S_{2}, S_{3} \in B_{K(X, Y)}$ and $T \in B_{L(X, Y)}$. It suffices to show that for any $\varepsilon>0$ there exists $K \in K(X, Y)$ such that

$$
\left\|S_{i}+T-K\right\| \leq 1+\varepsilon \quad(i=1,2,3) .
$$


Since $\bigcup_{i=1}^{3} S_{i}\left(B_{X}\right)$ has the compact closure in $Y$, there exists a finite subset $A$ of $I$ such that

$$
\left\|S_{i} x-P_{A} S_{i} x\right\|<\frac{1}{2} \varepsilon \text { for } x \in B_{x} \text { and } i=1,2,3 .
$$

By Theorem 2, choose a net $\left\{Q_{\alpha}\right\}$ in $B_{L(X, Y)}$, a net $\left\{K_{\alpha}\right\}$ in $K(X, Y)$ and $\alpha_{0}$ such that

$$
P_{A}\left(T-Q_{\alpha}\right)=0 \text { and }\left\|Q_{\alpha}-K_{\alpha}\right\|<\frac{1}{2} \varepsilon \text { for } \alpha \geq \alpha_{0}
$$

and

$$
\left\|\left(T-Q_{\alpha}\right) x\right\| \leq\|T x\| \text { for all } x \in X \text { and all } \alpha .
$$

Then for $x \in B_{X}, \alpha \geq \alpha_{0}$ and $i=1,2,3$, we have

$$
\begin{aligned}
\left\|S_{i} x+T x-K_{\alpha} x\right\| & \leq\left\|P_{A} S_{i} x+\left(T-Q_{\alpha}\right) x\right\|+\frac{1}{2} \varepsilon+\frac{1}{2} \varepsilon \\
& =\max \left\{\left\|P_{A} S_{i} x\right\|,\left\|\left(T-Q_{\alpha}\right) x\right\|\right\}+\varepsilon \\
& \leq \max \left\{\left\|S_{i} x\right\|,\|T x\|\right\}+\varepsilon \leq 1+\varepsilon .
\end{aligned}
$$

Thus $\left\|S_{i}+T-K_{\alpha}\right\| \leq 1+\varepsilon$ for $\alpha \geq \alpha_{0}$ and $i=1,2,3$.

Corollary 4. Let $X$ be a reflexive Banach space and let $Y$ be a closed subspace of $\left(\sum Z_{i}\right)_{c_{0}}\left(\operatorname{dim} Z_{i}<\infty\right)$. If either $X$ or $Y$ has the compact approximation property, then $K(X, Y)$ is an $M$-ideal in $L(X, Y)$.

Proof. Suppose $X$ has the compact approximation property. Let $0 \neq T \in L(X, Y)$ and let $K$ be a compact set in $X$. Then for any $\varepsilon>0$ there exists a compact operator $T_{1}$ on $X$ such that $\left\|T_{1} x-x\right\| \leq \varepsilon /\|T\|$ for all $x \in K$. Now $T T_{1} \in K(X, Y)$ and $\left\|T T_{1} x-T x\right\| \leq \varepsilon$ for all $x \in K$. This shows that $K(X, Y)$ is dense in $L(X, Y)$ in the topology of uniform convergence on compact sets in $X$. By Theorem $3, K(X, Y)$ is an $M$-ideal in $L(X, Y)$. The proof of the other case is similar.

\section{REFERENCES}

[1] E. M. Alfsen and E. G. Effros, Structure in real Banach spaces, Ann. of Math., 96 (1972), 98-173.

[2] E. Behrends, $M$-structure and the Banach-Stone Theorem, Lecture Notes in Mathematics 736, Springer-Verlag (1979).

[3] C.-M. Cho and W. B. Johnson, A characterization of subspaces $X$ of $l^{p}$ for which $K(X)$ is an $M$-ideal in $L(X)$, Proc. Amer. Math. Soc., 93 (1985), 466-470.

[4] $\longrightarrow M$-ideals and ideals in $L(X)$, J. Operator Theory, 16 (1986), 245-260.

[5] J. Diestel and J. Uhl, Vector measures, American Mathematical Survey, No. 15 (1977).

[6] A. Grothendieck, Produits tensoriels topologiques et espaces nucleaires, Mem. Amer. Math. Soc., No. 16 (1955). 
[7] P. Harmand and A. Lima, Banach spaces which are $M$-ideals in their biduals, Trans. Amer. Math. Soc., 283 (1983), 253-264.

[8] J. Hennefeld, A decomposition for $B(X)^{*}$ and unique Hahn-Banach extensions, Pacific J. Math., 46 (1973), 197-199.

[9] A. Lima, Intersection properties of balls and subspaces of Banach spaces, Trans. Amer. Math. Soc., 227 (1977), 1-62.

[10] - M-ideals of compact operators in classical Banach spaces, Math. Scand., 44 (1979), 207-217.

[11] J. Lindenstrauss and L. Tzafriri, Classical Banach Spaces I, Springer-Verlag, Berlin (1977).

[12] K. Saatkamp, M-ideals of compact operators, Math. Z., 158 (1978), 253-263.

[13] R. R. Smith and J. D. Ward, M-ideal structure in Banach algebras, J. Funct. Anal., 27 (1978), 337-349.

[14] R. R. Smith and J. D. Ward, Application of convexity and M-ideal theory to quotient Banach algebras, Quart. J. Math. Oxford (2), 30 (1979), 365-384.

[15] D. Werner, Remarks on M-ideals of compact operators, preprint.

Received March 15, 1988. Supported by Korea Science and Engineering Foundation grant No. 862-0102-009-2.

HANYANG UNIVERSITY

SEOUL 133-791, KoREA 